\title{
Adjusting Large-Scale Propagation Models for the Amazon Region Using Bioinspired Algorithms at 1.8 and $2.6 \mathrm{GHz}$ Frequencies
}

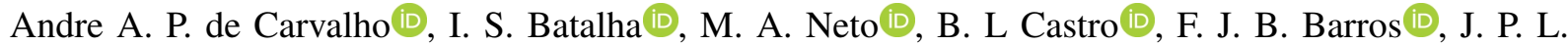 \\ Araujo (D), G. P. S. Cavalcante (iD) \\ Technology Institute, Telecommunication and Computation Laboratory, Federal University of Pará, Belém, \\ Pará,Brazil,andrepcarvalho@gmail.com, iurybatalha@gmail.com, miercio@ufpa.br,brunoslc21@gmail.com, \\ fjbbrito@gmail.com,jasmine@ufpa.br,gervasiogmeio@gmail.com
}

\begin{abstract}
In this work, a new method employs Bioinspired Computational (BIC) optimization from the genetic algorithm, bat algorithm, and flower pollination algorithm. Robust and accurate modeling of the input parameters adjusts the propagation models Stanford University Interim, Electronic Communication Committee, and Floating Interception that consider environments with characteristics specifically of urban regions in the Amazon. The lack of research related to the development of propagation models for Amazonian environments motivated this work. Thus, this application proves the effectiveness of using BIC techniques for modeling the communication channel. Measurement campaigns were carried out in the city of Belem, Brazil, for large-scale channel modeling on the frequencies of 1.8 and $2.6 \mathrm{GHz}$, belonging to the long-term evolution or fourth-generation mobile communications system (4G). After being adjusted by the optimum values calculated by the BIC techniques used, the models showed better results compared to modeling without optimization. Additionally, it was verified an error reduction of about $80 \%$ concerning the metrics root-mean-square error and standard deviation.
\end{abstract}

Index Terms - Channel Characterization, Measurement campaigns, Bioinspired Computational, Propagation Models.

\section{INTRODUCTION}

The great benefit that Long Term Evolution (LTE) brought to the new generation of networks was the increase in data traffic and, consequently, the provision of Quality of Service (QoS) to the customer. However, to satisfy the growing demand for high data consumption, the aggregation of the LTE cellular network with Wireless Local Area Network is the proposal that incorporates the 5G network [1]. Experts say the $4 \mathrm{G}$ and $5 \mathrm{G}$ need coexist for a while since the characteristics of these generations will help telecom operators operate both technologies simultaneously [2]. The LTE connection is the basis for 5G. In 2019, in the report of the company GSMA Intelligence, only $15 \%$ of the world population will be using $5 \mathrm{G}$ by 2025 [3].

Since LTE operates in different frequency bands, it is possible to activate carrier aggregation, that is, joining the connections of two frequencies operating only one carrier [4]. With this, the capacity of the available spectrum is increased, optimizing the network, and giving high speed to the end-user. Another important point is the signal coverage that is determined using propagation models. The models are mathematical tools developed with the objective of calculating the average behavior of the signals, the 
reason why it is necessary to know all the factors that influence the signal propagation of the mobile communication networks. Channel modeling is one of the factors that will provide the best efficiency of the mobile network, bringing high speed and increasing the number of users connected to the same transmission antenna. Therefore, some techniques using Bioinspired Computing (BIC) are great helpers for channel modeling. Through algorithms, the objective is to accelerate the optimization process, to solve complex optimization problems when compared to classic methods [5]-[9].

Therefore, this work proposes a method to find optimal values for the parameters of classic outdoor propagation models, since these models originate from environments with different characteristics from those found in the Amazon region. Using the Genetic Algorithm (GA), Flower Pollination Algorithms (FPA), and Bat Algorithm (BAT), the following parameters were adjusted: $(\gamma)$ representing the loss with the distance of the Stanford University Interim (SUI) model, $\left(A_{b m}\right)$ that defines the average loss of the Electronic Communication Committee (ECC) model and the parameters $\alpha$ and $\beta$ of the Floating Interception (FI) model. Thus, an accurate result is found for modeling in suburban outdoor environments in the Amazon region. Measurement campaigns were carried out at frequencies of 1.8 and 2.6 GHz to evaluate the applicability of the method. Power values from the Radio Base Station (ERB) selected along public roads in the city of Belém were collected. Four distinct routes were selected, representing the characteristic of the environment, which has the presence of vegetation.

The main contributions of this article are: (i) A measurement campaign in real environments (roads in the city of Belém, representing Amazonian environments); (ii) The use of BIC to optimize large-scale propagation models; (iii) Calculation of optimal values for models to better characterize the channel; (iv) Adjustments to the propagation model for the Amazon region; (v) Checking the best algorithm for adjusting the models, and (vi) A new methodology for the optimization of propagation models.

This study has seven sections. In Section II, there is an analysis of the similar works found in the literature. The methodology used in this work is in Section III. There is a description of the bioinspired algorithms used in this work in Section IV. In the following section, there is an explanation of the propagation models selected in this work. The results obtained in the proposed scenario are analyzed in Section VI, as well as presents the optimized parameters and the adjusted models, and Section VII presents the conclusion on the adjustments made.

\section{RELATED WORK}

Bioinspired computing emerges as a methodology for solving problems in several areas of knowledge, such as engineering, computer networks, security, etc. The following are some of the works found that use BIC to solve problems.

In [10], Alcantara applies a hybrid optimization technique using a regression neural network and a Cuckoo Search algorithm to solve problems in microwave applications. Used of Frequency Selective Surfaces (FSS) with triangular ring elements printed on fiberglass substrates. Was developed an FSSs with values bandwidth within the range of 8 to $12 \mathrm{GHz}$ and in a band-stop spatial filter has been synthesized, built, and measured. The results have shown that the technique generated good results when compared to the data measured. The technique was fast and accurate, being a viable option for the development of radio diffusion circuits, including planar FSS, filters, and resonators.

In [11] a bioinspired hybrid optimization technique is presented that associates a General Regression Neural Network with the Bat Algorithm, for the design and synthesis of FSS, its application in data 
communication systems by diffusion of millimeter waves, in the IEEE 802.15.3c standard. The projected device was of flat arrangements of metallizations, in the shape of a diamond. The FSS featured operation with ultra-wideband characteristics, its patch designed to cover a bandwidth of $30.0 \mathrm{GHz}$. The results proved to be fast and accurate, which consists of a more viable tool for the development of broadcasting circuits, including planar FSS, filters, and resonators.

In [12], was developed a BIC to synthesize the geometric dimensions of a diamond-shaped broadband FSS for filtering electromagnetic waves in satellite communication systems, for $\mathrm{X}(8-12 \mathrm{GHz})$ and $\mathrm{Ku}$ (12-18 GHz). A hybrid BIC technique was used, which combines the general regression neural network with a floral pollination algorithm. The optimization process was to tune the resonance frequency of the diamond FSS to $15.2 \mathrm{GHz}$. Thus, the results of an ultra-wideband framework were obtained with the most suitable dimensions to operate in the frequency bands of the $\mathrm{X}$ and $\mathrm{Ku}$ bands at the same time, making it applicable for operation in satellite communication systems.

In [13], Shahid proposed a particle swarm optimization to minimize the overall interference in the LTE network. A new Component Carrier (CC) selection method is proposed, aiming to converge to a stable value in hundreds of iterations. The results showed that the new proposed method presents better results when compared to traditional $\mathrm{CC}$ selection methods, i.e., random and round-robin in terms of throughput and fairness.

Shojafar proposed a new algorithm called P5G based on the particle swarm optimization. The main goal is to maximize the user's transfer rate or minimize the number of nodes used in the $5 \mathrm{G}$ network [14] . The results showed a reduction in the computational time and increased the user's transfer rate. Moreover, it was capable of better utilizing the network and computational resources.

Jayanthi proposed a new solution for the multi-path routing in Wireless Sensor Networks (WSN), in [15]. The design is focused on a structure that balances multiple performance metrics by combining a dynamic programming model of DNA sequence model with existent multi-path routing to find an optimized balanced routing sequence for real and non-real traffic. The algorithm aims to provide improved energy and throughput parameters. The results showed a significant improvement in the transfer and package delivery rates, and also a considerable reduction in the energy consumption when compared to the standard multi-path routing protocols.

In [16], in order to predict path loss at $800 \mathrm{MHz}$ and $2.600 \mathrm{MHz}$ frequencies, a hybrid model was developed using a combination of an artificial neural network and some propagation models. The results showed that the hybrid model based on error was more accurate than the other methods in the tested scenarios, presenting the lowest Root Mean Square Error.

In [17], a model for heterogeneous networks was created using artificial neural networks to predict the path loss for bands in frequencies ranging from $450 \mathrm{HMz}$ to $2.600 \mathrm{HMz}$, using only one formulation. Through a measurement campaign in Tunis - Tunisia, it was possible to validate the model. The results of the model demonstrated that the path loss prediction is efficient due to the absolute mean error approaching zero $\mathrm{dB}$, and the standard deviation (SD) of less than $7 \mathrm{~dB}$.

This work proposes to adjust parameters of the classical propagation models, through measured data, using bioinspired optimization algorithms. To the best of our knowledge, it is an innovative methodology, and it was not found in recent literature nor the use of optimization of the parameters of the propagation models through bioinspired algorithms and also applied in a environment as Amazon region. 


\section{Methodology}

The activities developed in this work were divided into four parts, as follows: measurement campaign, analysis of the data obtained, calculation of optimization of the parameters of the models using the BICs, and adjustments of the propagation models. Fig. 1 shows the flowchart of the methodology developed.

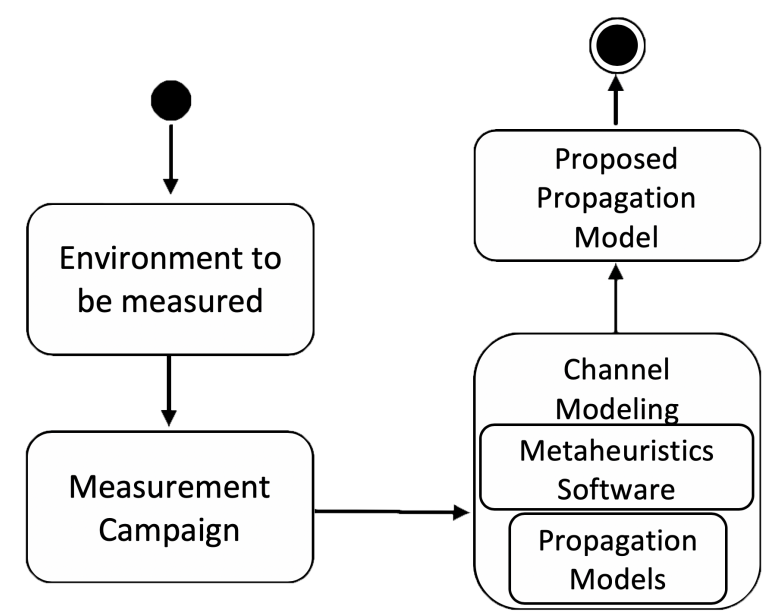

Fig. 1. Flowchart of the proposed methodology.

\section{A. Measurement Campaigns}

There were measurement campaigns in the streets of the city of Belém, in Brazil, prioritizing roads with predominant afforestation. It is important to note that the city is close to the Equator and does not have four clearly defined seasons during the year. Therefore, the city of Belém has only two predominant periods: with little rain and another period with a lot of rain.

The city of Belém has a total area of $1,064.918 \mathrm{~km}^{2}$, and its altitude is ten meters above sea level. Fig. 2(a) provides an aerial view of the city, which has many buildings in the city center and the absence in the area considered to be on the periphery, while Fig. 2(b) with the presence of tall canopy trees creating tunnels of trees, forming a unique environment. In measurement campaigns, the

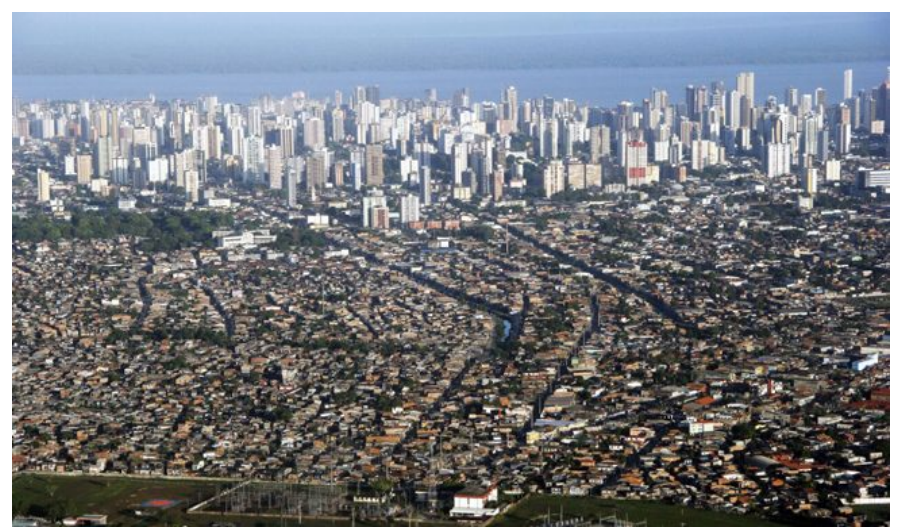

(a)

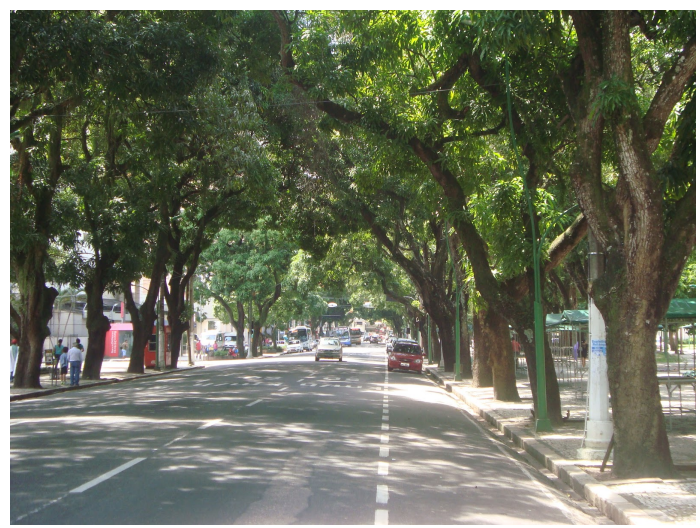

(b)

Fig. 2. City of Belém.

G-NetTrack application obtained the data. This application uses GPS (Global Positioning System) to 
locate the measured points and generates data in a text file, with information on communication quality at signal levels. Therefore, G-NetTrack is used to monitor mobile networks and deliver the results of network evaluation metrics. The application shows basic metrics in real-time, as well as graphs with recent historical records of the collected metrics [18] [19].

A Mi 5s Plus smartphone (Xiaomi) was used for data collection, and among its main features are Quad-Core Max 2.3 GHz processor, 6 GB of RAM, 128 GB of internal memory, Kernel 3.18.20, and Operating System Android 6. G-NetTrack is a software used to measure the signal levels of a given wireless communication network already implemented, evaluating the quality of service to users of the channel.

For the treatment and processing of data, implementation of algorithms to adjust the selected models, a computer was used with the following configuration: Intel i7 processor, 16 GB of RAM, 512 HD SSD, and iOS operating system using MatLab® development tool.

The smartphone with the G-NetTrack was at $1.6 \mathrm{~m}$ on a fixed rod. The rod was inside a car, which has a constant speed of $30 \mathrm{~km} / \mathrm{h}$ along the routes. Fig. 3 shows the arrangement of the equipment in the data collection.

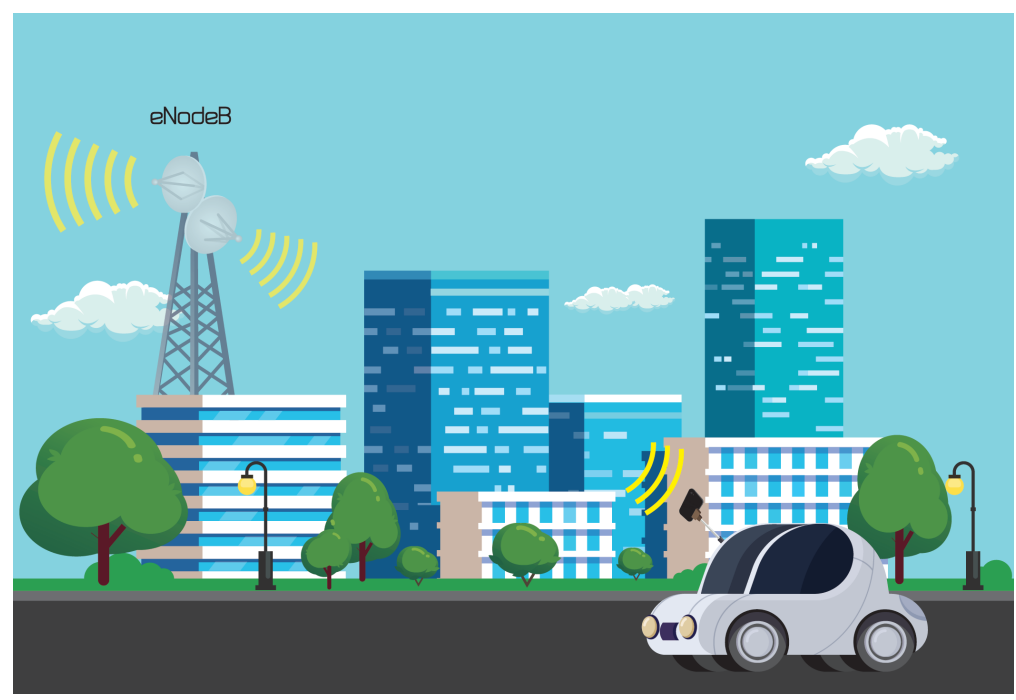

Fig. 3. Methodology measurement campaigns.

During the measurement campaign, it was possible to map the data transmitting antennas (eNodeB) installed by one of the mobile phone operators in the city. The power data received from the transmitted signal at $1.800 \mathrm{MHz}$ and $2.600 \mathrm{MHz}$ were stored. The antennas selected in the routes have characteristics seen in Table I.

TABLE I. PROPERTY OF ANTENNAS OF ROUTES

\begin{tabular}{cccccc}
\hline Route & Power & \multicolumn{2}{c}{ Gain } & \multicolumn{3}{c}{ Height Frequency Environment } \\
\hline 1 & 40 Watts & $15.4 \mathrm{dBi}$ & $40 \mathrm{~m}$ & $2.6 \mathrm{GHz}$ & Suburban \\
2 & 81.8 Watts & $15.4 \mathrm{dBi}$ & $50 \mathrm{~m}$ & $1.8 \mathrm{GHz}$ & Urban \\
3 & 40 Watts & $15.4 \mathrm{dBi}$ & $40 \mathrm{~m}$ & $2.6 \mathrm{GHz}$ & Suburban \\
4 & 81.8 Watts & $15.3 \mathrm{dBi}$ & $50 \mathrm{~m}$ & $1.8 \mathrm{GHz}$ & Suburban \\
\hline
\end{tabular}

It is worth mentioning that the routes were selected according to criteria of tree tunnels (Fig. 2(b)) and buildings around the streets (Fig. 2(a)), obtaining a mix construction and afforestation scenario to observe the performance of the signal received by the user. 
Fig. 4 shows the geographical positions of each antenna, along with the measured points and received power levels.

With the received power data, BICs were used to optimize some essential parameters in the models to adjust the models with the unique characteristics of the environment.

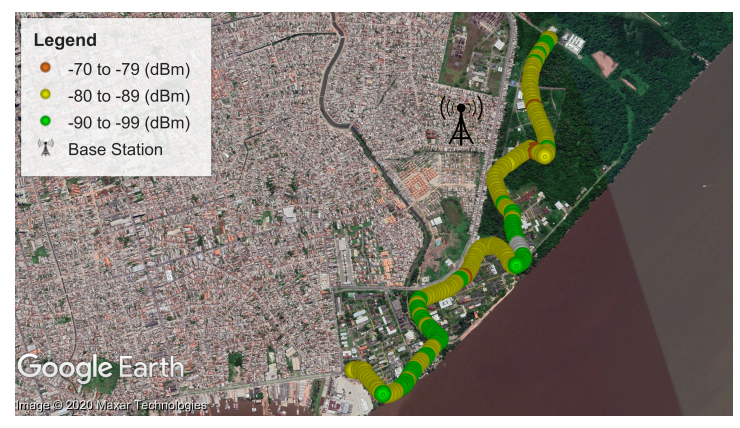

(Route 1)

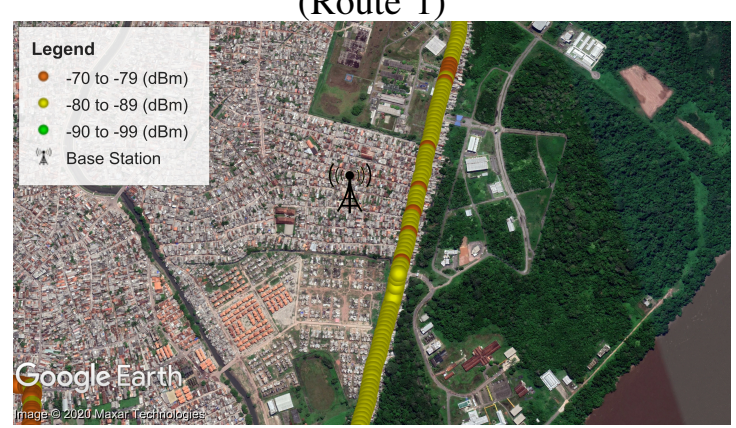

(Route 3)

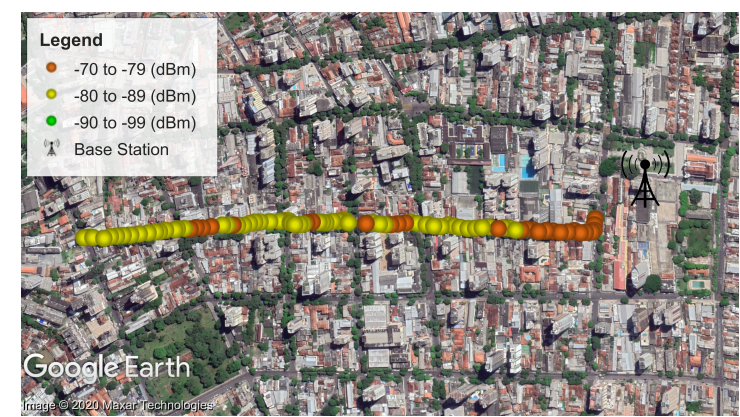

(Route 2)

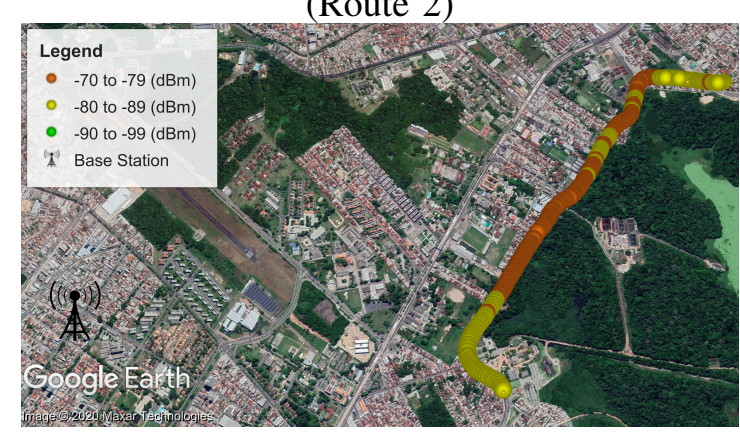

(Route 4)

Fig. 4. Measurement routes.

\section{Computational SteP}

Bioinspired computing (BIC) has aroused great interest among researchers as they are powerful methods to tackle a series of engineering and industrial problems, such as the search for optimization concerning cost and energy consumption, improving network performance and efficiency [20], [21]. Some algorithms stand out, such as Genetic algorithms [22], Artificial neural networks [23], Particle swarm optimization [24], Flower pollination algorithm [25], and the BAT algorithm [26] and the Cuckoo Search algorithm [27].

The optimization process involves making an objective assessment of the function through an extensive iterative procedure. Advanced computational tools usually made these assessments, which means that efficient optimization requires an efficient simulator.

There are several ways to solve optimization dilemmas. However, the best-known formulation is to write a problem with nonlinear optimization, as expressed below equation 1 .

$$
\begin{aligned}
& \text { minimize }_{i}(x), i=1 ; 2 ; \ldots ; M \\
& \text { subject to restrictions } h_{j}(x), j=1,2, \ldots, J \\
& \text { and } g_{k}(x) \leq 0, k=1,2, \ldots, K
\end{aligned}
$$

Where $f_{i}, h_{i}$ and $g_{k}$ are generic nonlinear functions. The vector project $x=\left(x_{1}, x_{2}, \ldots, x_{n}\right)$ can be a continuous, discrete, or mixed inn-dimensional space. The $f_{i}$ functions are called cost or objective functions, and when $M>1$ optimizes multi-objective or multi-criteria [26]- [28]. Multiple objectives 
can be combined into a single objective, although multiple objectives can return much more information about the problem than necessary. Additionally, the equation described above represents a minimization problem. This problem can also be a maximization problem, just replacing $f_{i}(x)$ by $-f_{i}(x)$.

The use of BIC with increased efficiency is because these algorithms simulate the best resources of nature, especially selection based on the principle of survival of the fittest in biological systems that have evolved by natural selection over millions of years.

Two essential characteristics of metaheuristics are intensification and diversification [29]. Intensification does local and intensive research, while diversification ensures that the algorithm exploits the entire research space on a global scale. A suitable balance between the two resources is very considerable for the efficiency and performance of a given algorithm.

The fact that BICs efficiently solve arbitrary optimization problems and are generally self-organizing, adaptable, and tolerant of random events [30] explains the growing interest shown by engineering researchers. Additionally, BICs can provide solutions to real classification problems, such as proximity, vision, pattern recognition, identification, and control, as well as rigorous analysis of unique applications.

Stochastic optimization studies where the constraint or the parameters depend on random variables, the metaheuristics are part of the stochastic group, applying algorithms or techniques that use some level of randomness to find suitable solutions to difficult problems [29]- [31].

The selected algorithms use random values to initialize the solution population. However, the maximum and minimum values obtained in real measurements limit these random values, to allow a fair comparison between the algorithms.

In this study, there is the use of the number of iterations as a stopping criterion. Therefore, every time in an algorithm execution, it may present differences in the optimized parameters, due to the diversity found in bioinspired, and the algorithm stores the best solution after the maximum number of iterations. In this work, there is the use of the Genetic Algorithm, the Flower Pollination Algorithm (FPA), and the Bat Algorithm (BAT).

\section{A. Genetic Algorithm}

Using the AG available in MATLAB by function gaoptimset was used as input parameters: standard deviation (SD) of $10 \%$ concerning the database collected in the measurement, a population with a size of 100 individuals, a maximum number of 1000 generations, and with a limit of $0,05 * n_{\text {generation }}$ (so as not to have many generations without progress).

\section{B. Bat Algorithm}

This algorithm is based on the echo behavior of bats to determine the distance and position of obstacles using ultrasonic waves and to analyze the time spent by the waves emitted to reflect on the objective and return to the emission source in the form of an echo [26].

The variation in amplitude and the pulse emission rate makes the control and diversification of the algorithm. BAT initially generates a population of random solutions, called a group. The characterization of each member of this group is assigned a $v_{i}$ speed, $x_{i}$ position, and a pulse emission frequency $f$.

In algorithm operation, there is the update frequency, speed, and position of each particle. $\beta \in[0,1]$ is a vector of random numbers with uniform distribution. The frequency will have a random value 
within a uniformly distributed range $\left[f_{\min }, f_{\max }\right]$, and $X$ will be the best solution found until the current iteration, after buying all the resolutions between all bats.

The algorithm can perform a local search by comparing the amount of a random number $r a n d \in[0,1]$ with the value of the pulse emission rate $r_{i}$. If rand $>r_{i}$, new search will be carried out around the best current solution. The average pulse emission amplitude of all bats is called $A_{i}$ and updated for each iteration. The choice of $f_{\min }$ and $f_{\max }$ requires experience because initially, each bat can have different amplitudes and pulse emission rate values, achieved due to random initialization.

\section{Flower Pollination Algorithm}

The Flower Pollination Algorithm (FPA) [25] is inspired in the pollen transference process (pollination) common in flowers. There are two types of pollination: biotic and abiotic. In the biotic type, the pollen is transferred by a pollinator such as insects and animals. The abiotic type, on the other hand, does not require a pollinator and the pollen is transported by other means such as wind and water. The FPA uses the biotic process to calculate the suitability of the solutions. The code heuristic assumes that each plant has only one flower and each flower produces only one gamete. The algorithm implements two types of pollination: global and local.

The algorithm begins by randomly generating a population of flowers and, subsequently, the best solution in the initial population is selected. The odds ratio is used to choose whether the pollination applied is global or local. That is how the flower evolution happens throughout time and search space [25].

\section{LARGE-ScAle Propagation Models}

In telecommunications, propagation models are used to calculate the behavior of signal in different environments [32]. The models use equation to accurately predict the large-scale propagation loss curves, which have logarithmic behavior. Also, used are parameters that try to describe the specific loss behavior for obstructions, something not observed in free space, such as buildings, vegetation, polarization, height, and situations without direct aim [8].

Large-scale modeling studies analyze the average behavior of the loss of propagation or power received with the distance and in the variability of the signal strength of a specific location. In this article, we will be the use of the classic large-scale models SUI, ECC-33, and Floating Intercept.

\section{A. Stanford University Interim Propagation Model}

A joint project with Stanford University and the 802.16 IEEE group, after extensive work, to design a channel model for networks in suburban environments, resulting in the Stanford University Interim (SUI) propagation loss model [33]. In this model, there is a categorization of the median loss of trajectory in three different environments, called A, B, and C. Type A environments are associated with a maximum path loss, used for regions with moderate to intense vegetation and very rugged relief. Type $B$ is associated with the intermediate terrain between types A and C, with light vegetation density. Finally, type $\mathrm{C}$ is suitable for regions with minimal loss of travel. According to in (2), it is possible to determine the path loss of the SUI model.

$$
L=A+10 \gamma \log \left(\frac{d}{d_{0}}\right)+X_{f}+X_{h}+s
$$

Brazilian Microwave and Optoelectronics Society-SBMO Brazilian Society of Electromagnetism-SBMag received 23 Oct 2020; for review 9 Nov 2020; accepted 25 Mar 2021 (C) 2021 SBMO/SBMag (cc) BY ISSN 2179-1074 
Journal of Microwaves, Optoelectronics and Electromagnetic Applications, Vol. 20, No. 3, September 2021 DOI: http://dx.doi.org/10.1590/2179-10742021v20i31099

$$
\begin{gathered}
A=20 \log \left(\frac{4 \pi d_{0}}{\gamma}\right) \\
\gamma=a-b h_{b}+\frac{c}{h_{b}} \\
X_{f}=6 \log \left(\frac{f}{2000}\right) \\
X_{h}=-10.8 \log \left(\frac{h_{r}}{2}\right)
\end{gathered}
$$

where:

$A$ is the loss in free space in (3);

$\gamma$ represents the exponent of path loss and is related to the environment in (4);

$d$ is the distance between terminals in meters;

$d_{0}$ is the initial distance of $100 \mathrm{~m}$;

$h_{b}$ is the height of the base station in meters $\left(10 m<h_{b}<80 m\right)$;

$s$ is a path loss factor with characteristics of a normal distribution that considers the shading of trees and structures;

$X_{f}$ is the correction factor for the transmitting antenna frequency in (5);

$X_{h}$ is the correction factor for the receiving antenna in (6); in types A and B;

The factors $a, b$ and $c$ depending on the environment under analysis, standardized according to Table II [34].

TABLE II. MODEL CONSTANTS FOR IEEE 802.16 MODEL FOR 2.5-2.7 GHz BAND

\begin{tabular}{cccc}
\hline Model constant Terrain Type A & Terrain Type B Terrain Type C \\
\hline a & 4.6 & 4.0 & 3.6 \\
b & 0.0075 & 0.0065 & 0.005 \\
c & 12.6 & 17.1 & 20 \\
\hline
\end{tabular}

\section{B. Electronic Communication Committee Propagation Model}

The Electronic Communication Committee (ECC-33) model corresponds to an extrapolation of the measurements made by Okumura in Tokyo [35], where the classification of large and medium-sized cities was used, with correction factors for suburban or open areas. The path loss for the ECC-33 model, in $\mathrm{dB}$, is given by (7).

$$
\begin{gathered}
L=A_{f s}+A_{b m}-G_{b}-G_{r} \\
A_{f s}=92.4+20 \log (d)+20 \log (f) \\
A_{b m}=20.41+9.83 \log (d)+7.894 \log (f)+9.56[\log (f)]^{2} \\
G_{b}=\log \left(\frac{h_{b}}{200}\right)\left\{13.958+5.8\left[\log (d)^{2}\right]\right\}
\end{gathered}
$$

where:

$A_{f s}$ is the loss in free space in $\mathrm{dB}$ in (8);

$A_{b m}$ is the loss of average travel in $\mathrm{dB}$ in (9);

$d$ is the distance in $\mathrm{km}$; 
$f$ is the operating frequency in $\mathrm{GHz}$;

$G_{b}$ is the gain factor by the height of the base station in (10) that depends on $h_{b}$;

$G_{r}$ is the gain factor for the height of the receiving antenna that depends on $h_{r}$.

For medium-sized cities, $G_{r}$ is given by in (11), and for large cities, it is given by in (12):

$$
\begin{gathered}
* G_{r}=[42.57+13.7 \log (f)]\left[\log \left(h_{r}\right)-0.585\right] \\
* * G_{r}=0.759 h_{r}-1.862
\end{gathered}
$$

\section{Floating-Intercept Model}

The Floating-Intercept FI is a model part of WINNER II and 3GPP standards (3rd Generation of the Public-Private Partnership) [36]- [37]. It is a single frequency model with two parameters and it does not consider a physical anchor based on the transmitted power. FI is expressed in (13).

$$
P L^{F I}(d)=\alpha+10 \beta \log _{10}(d)+X_{\sigma}^{F I}
$$

the parameter an is the $\mathrm{FI}$ in $\mathrm{dB}$, and $\beta$ is the slope. $X_{\sigma}^{F I}$ is the Gaussian shadowing with zero average value in $\mathrm{dB}$ and it describes large-scale signal fluctuations on average propagation loss over distance. The Least Squares Method is used to turn the parameters of $a$ and $\beta$ minimizing the standard deviation (SD) $\sigma$. FI model can be used to frequencies above $6 \mathrm{GHz}$ as $5 \mathrm{G}$ frequencies, for example [9]. In [38][39], FI models present shading standard deviations similar in mmWave channels outdoors. FI model also can be used for multi-frequency model and it is called ABG model [40], but if it will be used for single frequency the parameter $(\gamma)$, which is frequency dependent, must be set 0 or 2 .

\section{Parameter Optimization}

After data collection and treatment, there was a selection of some parameters to optimize and adjust the models mentioned in the previous section. The three BICs in this work (the Genetic Algorithm (GA), Flower Pollination Algorithm (FPA), and Bat Algorithm (BAT)) optimized the chosen parameters.

In the SUI model, the parameters chosen for optimization are $\gamma$, which according to in (4), depends on three variables: $a, b$ and $c$, well as the frequency parameter and the weight of $X_{f}$ in (5), referring to the scalar multiplied by the logarithm and which depends on the frequency of the transmitting antenna, in this work we adopted to call it $x$. The $\gamma$ parameter is the exponent of path loss that will determine the loss to the distance.

In the ECC-33 model, the optimized parameter was $A_{b m}$, which represents the average path loss of over the measured data in (9). Each of the previous values is the weights of the equation and were replaced by variables to be optimized. The optimized variables refer to distance and frequency, as the frequencies used in this study do not fit the model, so it would be necessary to optimize these parameters. With the substitution of the values of in (14), we have (15), wherein each variable is a parameter to be optimized using BIC.

$$
\begin{gathered}
A_{b m}=20.41+9.83 \log (d)+7.83 \log (f)+9.56\left[\log (f)^{2}\right] \\
A_{b m}=x_{1}+x_{2} \log (d)+x_{3} \log (f)+x_{4}\left[\log (f)^{2}\right]
\end{gathered}
$$


Finally, the parameters chosen to optimize the FI model were $\alpha$ and $\beta$ (13), which represent the intercept coefficient and the slope of the line, respectively. And, the Table III shows all the parameters chosen for optimization to their respective propagation models.

TABLE III. OPTIMIZED PARAMETERS WITH BICs

\begin{tabular}{lllll}
\hline Models & \multicolumn{4}{c}{ Parameters } \\
\hline SUI & $a$ & $b$ & $c$ & $x$ \\
ECC-33 & $x_{1}$ & $x_{2}$ & $x_{3}$ & $x_{4}$ \\
FI & $\alpha$ & $\beta$ & - & - \\
\hline
\end{tabular}

In the next section, there will be a presentation of the optimized values of the parameters and the adjusted models. Also, there will be a comparison between the classic models and adjusted models.

\section{RESULTS}

In this section, there is an explanation and presentation of the optimized values for model adjustments. Also shown will be the models performed comparing them with classic models and adjusted models, resulting in three adjustments for each model in each route.

Finally, it is essential to explain that the graphs presented in this section show maximum values of the average distance of 800 meters because the measurement was in a real environment, and it was not possible to control the transmitter.

\section{A. Optimized Parameters}

The adjusted parameters are calculated by the equations in (4), (5), (9) and (13). The parameters $\gamma$ and $X_{f}$ are in the SUI model in (4) and (5), respectively. The parameter $A_{b m}$ is in the model ECC-33 in (15), and the parameters $\alpha$ and $\beta$ are given in the model FI in (13).

For all algorithms, the optimization indexes could vary between the minimum and maximum limits, physically possible for the models studied. For this reason, the parameters found for each route reached almost similar values. The results are presented below in Table IV, V, and VI, according to the selected propagation model, RMSE metrics calc, and standard deviation (SD).

\section{B. Analysis of Results}

When analyzing these results found in the optimization of the SUI propagation model, it is observed that for all the algorithms (AG, BAT, and FPA) there were significant adjustments for the presented routes. In Table IV, it is possible to see that for the RMSE metric it obtained an optimization of $66 \%$ for Route $1,40 \%$ for Route 2, 68\% for Route 3, and 50\% for Route 4 . Route 3 highlights the best optimization result found. Thus, it is noted that the propagation model in its classic version does not apply to the measured data. For all routes, it is possible to check the adequacy of the data after optimization. 
TABLE IV. RESULTS PARAMETERS SUI

\begin{tabular}{llcccccc}
\hline \multicolumn{2}{c}{ SUI } & a & b & c & x & RMSE & SD \\
\hline & Literature & 4 & 0.0065 & 17.1 & 6 & 22.08 & 6.44 \\
Route 1 & AG & 6.65 & 0.0001 & 14.45 & 10 & 7.46 & 5.28 \\
& BAT & 6.47 & 0.0001 & 21.53 & 10 & 7.46 & 5.28 \\
& FPA & 7.59 & 0.0341 & 31.69 & 10 & 7.46 & 5.28 \\
\hline \multirow{5}{*}{ Route 2 } & Literature & 4 & 0.0065 & 17.1 & 6 & 14.30 & 5.71 \\
& AG & 9.74 & 0.1056 & 48.25 & 1 & 8.46 & 4.90 \\
& BAT & 4.85 & 0.0001 & 28.78 & 1 & 8.46 & 4.90 \\
& FPA & 8.28 & 0.0615 & 11.09 & 1 & 8.46 & 4.90 \\
\hline \multirow{5}{*}{ Route 3 3} & Literature & 4 & 0.0065 & 17.1 & 6 & 23.30 & 5.17 \\
& AG & 7.58 & 0.0201 & 22.98 & 2.11 & 4.48 & 3.22 \\
& BAT & 6.59 & 0.0048 & 33.58 & 9.10 & 4.48 & 3.22 \\
& FPA & 8.38 & 0.0437 & 28.45 & 2.11 & 4.48 & 3.22 \\
\hline \multirow{5}{*}{ Route 4 4} & AG & 5.75 & 0.0065 & 17.1 & 6 & 15.87 & 5.42 \\
& LAterature & 4 & 0.001 & 18.22 & 1 & 7.94 & 4.52 \\
& BAT & 5.29 & 0.0001 & 36.40 & 1 & 7.94 & 4.52 \\
& FPA & 5.30 & 0.0043 & 42.85 & 1 & 7.94 & 4.52 \\
\hline
\end{tabular}

When analyzing the results found in the optimization with the propagation model ECC-33, the algorithms optimized the model in order to represent the measured data. In Table V, it is possible to see that for the RMSE metric it obtained an optimization of 39\% for Route1, 52\% for Route 2, 52\% for Route 3, and $14 \%$ for Route 4 . Route 2 and 3 stand out as the best optimization result found. For all routes, it is possible to check the adequacy of the data after optimization.

TABLE V. RESULTS PARAMETERS ECC-33

\begin{tabular}{llcccccc}
\hline \multicolumn{2}{c}{ ECC-33 } & $\boldsymbol{x}_{\boldsymbol{1}}$ & $\boldsymbol{x}_{\boldsymbol{2}}$ & $\boldsymbol{x}_{\boldsymbol{3}}$ & $\boldsymbol{x}_{\boldsymbol{4}}$ & RMSE & SD \\
\hline & Literature & 20.41 & 9.83 & 7.894 & 9.56 & 10.87 & 4.95 \\
Route 1 & AG & 33.36 & 10 & 2.840 & 0.78 & 6.62 & 4.18 \\
& BAT & 23.11 & 10 & 1.810 & 8.50 & 6.62 & 4.18 \\
& FPA & 25.65 & 10 & 2.350 & 6.87 & 6.62 & 4.18 \\
\hline \multirow{5}{*}{ Route 2 } & Literature & 20.41 & 9.83 & 7.894 & 9.56 & 8.13 & 3.19 \\
& AG & 28.33 & 1.27 & 6.910 & 1.80 & 3.87 & 2.54 \\
& BAT & 23.62 & 1.27 & 7.620 & 7.12 & 3.87 & 2.54 \\
& FPA & 24.51 & 1.27 & 3.810 & 1.48 & 3.87 & 2.54 \\
\hline \multirow{5}{*}{ Route 3 3} & Literature & 20.41 & 9.83 & 7.894 & 9.56 & 12.09 & 5.25 \\
& AG & 27.49 & 10 & 2.662 & 7.31 & 5.79 & 3.31 \\
& BAT & 35.59 & 10 & 2.630 & 0.37 & 5.79 & 3.31 \\
& FPA & 21.38 & 10 & 0.140 & 9.98 & 5.79 & 3.31 \\
\hline \multirow{5}{*}{ Route 4 4} & Aiterature & 20.41 & 9.83 & 7.894 & 9.56 & 5.50 & 3.01 \\
& AG & 24.73 & 6.17 & 9.810 & 9.31 & 4.71 & 2.68 \\
& BAT & 20.13 & 6.17 & 2.700 & 4.64 & 4.71 & 2.68 \\
& FPA & 28.76 & 6.17 & 9.260 & 4.82 & 4.71 & 2.68 \\
\hline
\end{tabular}

When analyzing the results found in the optimization with the FI propagation model, which have only two parameters to be adjusted, different from the SUI and ECC-33 model, even so, the algorithms 
TABLE VI. RESULTS PARAMETERS FI

\begin{tabular}{llcccc}
\hline & FI & $\boldsymbol{\alpha}$ & $\boldsymbol{\beta}$ & RMSE & SD \\
\hline & Literature & 17.6 & 3.3 & 18.95 & 6.11 \\
Route 1 & AG & 21.19 & 3.82 & 6.54 & 4.18 \\
& BAT & 44.56 & 2.96 & 6.61 & 4.18 \\
& FPA & 21.02 & 3.83 & 6.54 & 4.18 \\
\hline & Literature & 17.6 & 3.3 & 9.49 & 4.41 \\
Route 2 & AG & 63.91 & 1.92 & 3.80 & 2.51 \\
& BAT & 64.11 & 1.91 & 3.80 & 2.51 \\
& FPA & 64.14 & 1.91 & 3.80 & 2.51 \\
\hline & Literature & 17.6 & 3.3 & 20.38 & 5.37 \\
Route 3 & AG & 1 & 4.62 & 4.99 & 3.07 \\
& BAT & 17.36 & 4.03 & 5.21 & 3.12 \\
& FPA & 1 & 4.62 & 4.99 & 3.07 \\
\hline & Literature & 17.6 & 3.3 & 11.23 & 4.51 \\
Route 4 4 & AG & 51.82 & 2.36 & 4.76 & 2.70 \\
& BAT & 52.77 & 2.34 & 4.76 & 2.70 \\
& FPA & 52.77 & 2.35 & 4.76 & 2.70 \\
\hline
\end{tabular}

needed to optimize the model in order to represent the measured data. In Table VI, it is possible to see that for the RMSE metric it obtained an optimization of $65 \%$ for Route1, 59\% for Route 2, 75\% for Route 3, and 57\% for Route 4. Route 3 highlights the best optimization result found.

It is important to note, to analyze the graphs in this section, that the AG, BAT, and FPA algorithms, for all routes, have similar results in the proposed modeling. Thus, when plotting the lost path data, the graphical representations with AG (yellow line), BAT (blue line), and FPA (green line) are superimposed. However, it is possible to state that, in all cases, the RMSE data after optimization, obtained similar behavior.

Fig. 5 shows all routes with optimization for the propagation models (SUI, ECC, and FI). Comparing the classical modeling (in red) and the adjusted modeling, Fig. 5(a) with a frequency of $2.6 \mathrm{GHz}$, it is possible to verify the convergence of the adjusted model with the measured data from approximately 400 meters away. In the second route, Fig. 5(b), with a frequency of $1.8 \mathrm{GHz}$, the proposed model follows the trend of the data measured, after $500 \mathrm{~m}$ away from the antenna. On Route 3, with $2.6 \mathrm{GHz}$ in Fig. 5(c), after optimization, the modeling begins to converge with the data measured after $400 \mathrm{~m}$, similar to the modeling on Route 1. Finally, on path 4, with a frequency of $1.8 \mathrm{GHz}$, Fig. 5(d), the convergence of the modeling starts after about $300 \mathrm{~m}$ between the transmitting antenna and receiving equipment.

The obstructions caused by trees and buildings directly influence the attenuation of the signal received by the user. According to the calculation of the path loss coefficient, the higher its value, the more obstructed the environment. In this context, Fig. 6 illustrates the measured data with the four routes of the LTE system and shows the random variations of the average path loss (in $\mathrm{dB}$ ) caused by shading of the woody environment.

Where the dashed black lines represent the modeling using the basic loss due to free space, with different Path Loss Exponent (PLE) values, and the red line represents the modeling with the free space 

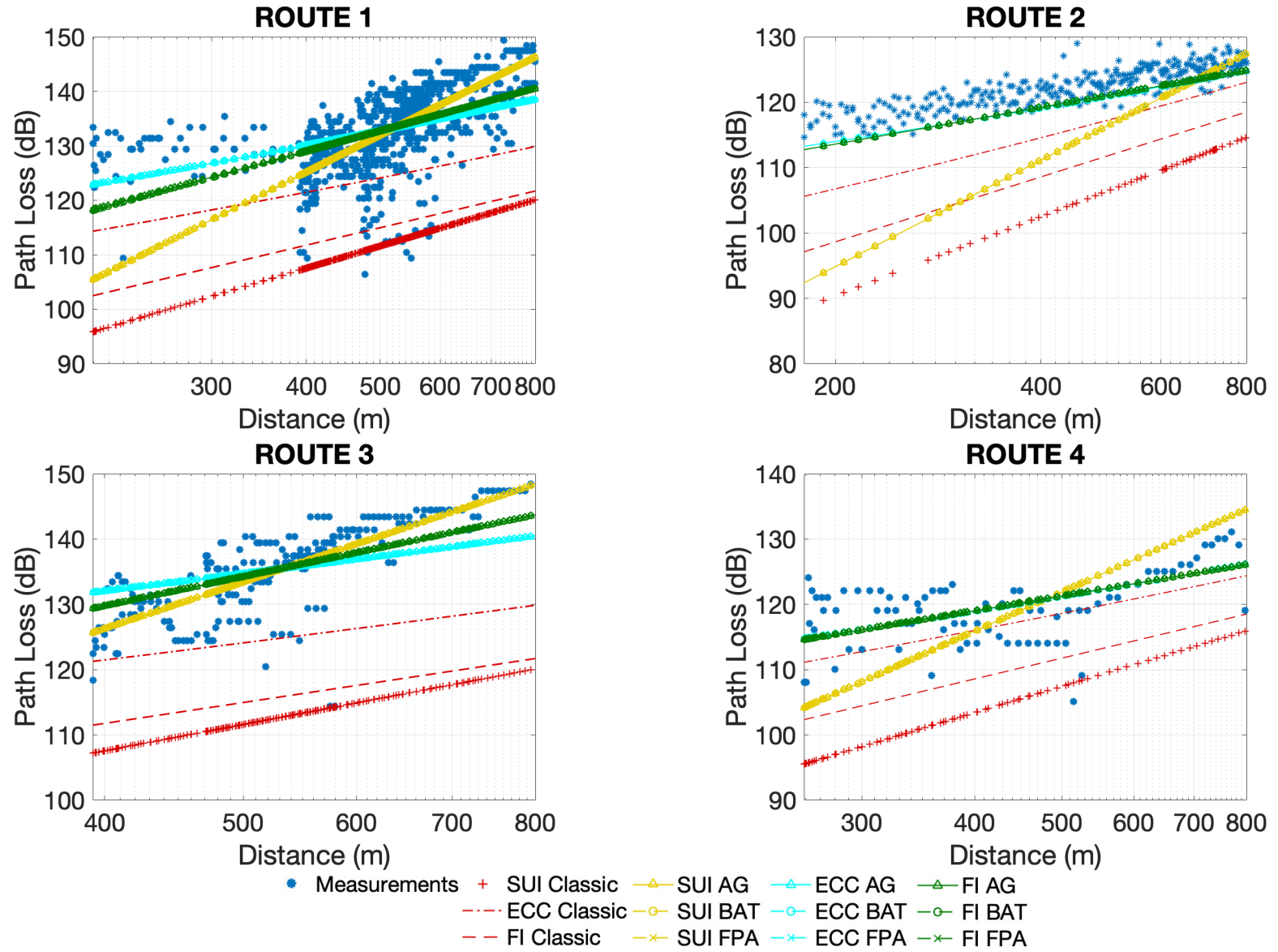

Fig. 5. Optimization results.

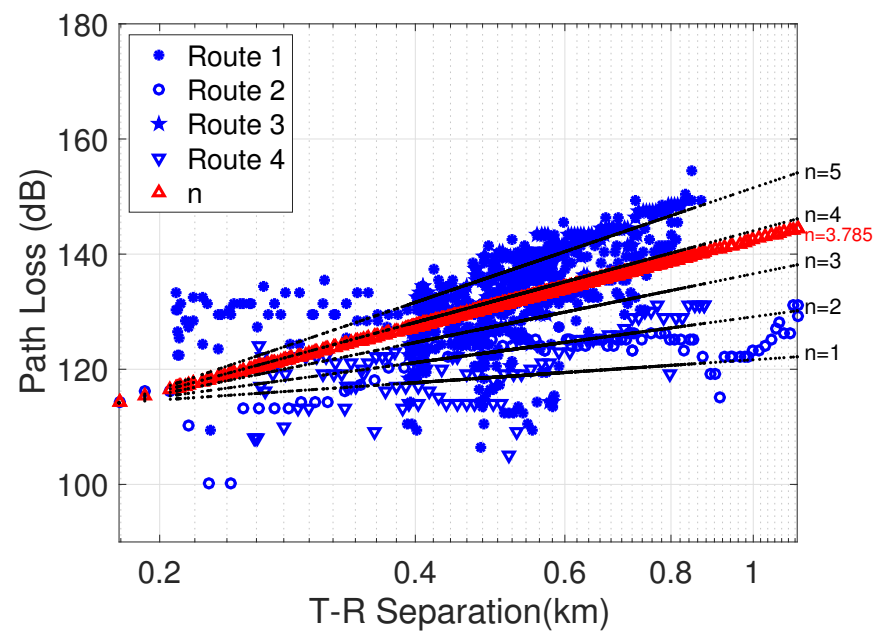

Fig. 6. Scatter plot of measured data and path loss exponent for routes in an arboreous environment.

model with the proposed PLE value. It is also possible to observe that the measured initial loss value is $116 \mathrm{~dB}$, and the loss value calculated with the free space model is $114.2 \mathrm{~dB}$. Thus, the initial loss value obtained by linear regression is very close to the value calculated with the free space model, and this result reduces the deviation between the measured data and the data calculated in the free space loss model, returning a value for $3.785 \mathrm{~dB}$ PLE and $7.65 \mathrm{~dB}$ standard deviation. 
Fig.7 presents the modeling using the free-space model with PLE values from the literature ( $P L E=$ $2)$ and the proposed PLE value $(P L E=3.78)$.

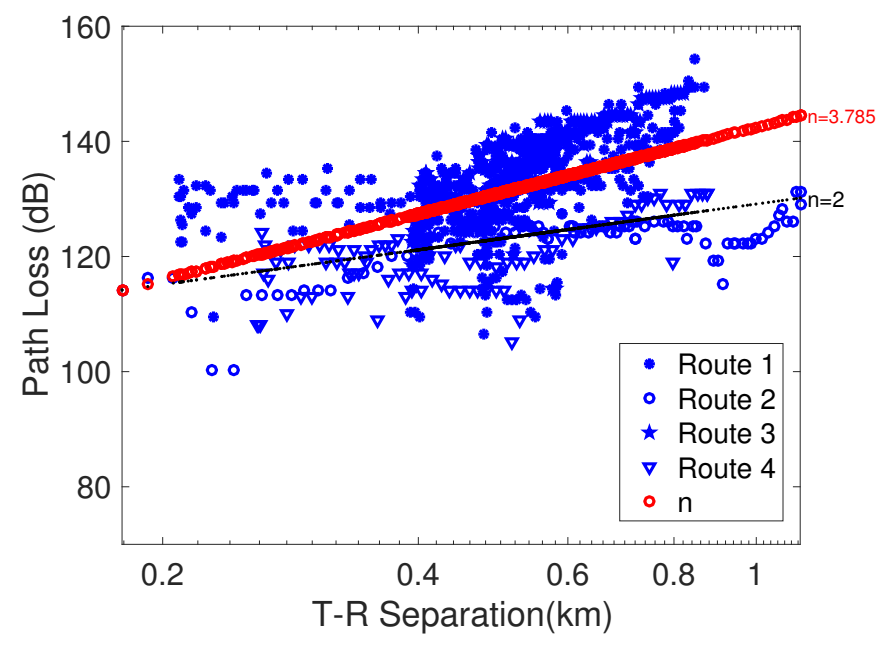

Fig. 7. Modeling with the free-Space model adopting values of classic PLE and proposed PLE.

This result shows that it is an environment obstructed by buildings above 3 floors, residences below 3 floors, dense vegetation, high flow of cars and people, a characteristic inherent to the Amazon region, justifying the choice of the routes under study.

This work addresses the need for parameter optimization to characterize the frequency channel studied with the models chosen to monitor the data found for the Amazon region.

With these results, it is possible to consider [16] that it presented RMSE values for the $2.6 \mathrm{GHz}$ frequency from 9.09 to $10.33 \mathrm{~dB}$ on two routes in suburban environments. It is also possible to analyze the standard deviation values of the models in [41], which ranged from 7.63 to $9.77 \mathrm{~dB}$ in environments that do not take vegetation and constructions into account. In [17] , the authors showed accuracy results, with a mean absolute error close to zero $[\mathrm{dB}]$ and a standard deviation of less than $7 \mathrm{~dB}$, but in environments similar to [41]. For the $1.8 \mathrm{GHz}$ frequency, there is the work [42] that presents values of $6.84 \mathrm{~dB}$ and $3.7 \mathrm{~dB}$, for RMSE and Standard Deviation, respectively. The studies cited were carried out in different environments than the study in this work.

When analyzing the behavior of the algorithms, through Fig. 8, for the SUI model, it is possible to identify that the AG has the worst performance when finding the best result of RMSE. For Route 1, Fig. 8 (a), for AG, it was necessary more iterations to converge to the optimal solution, while for BAT, it more quickly, and FPA had a better performance than AG. 

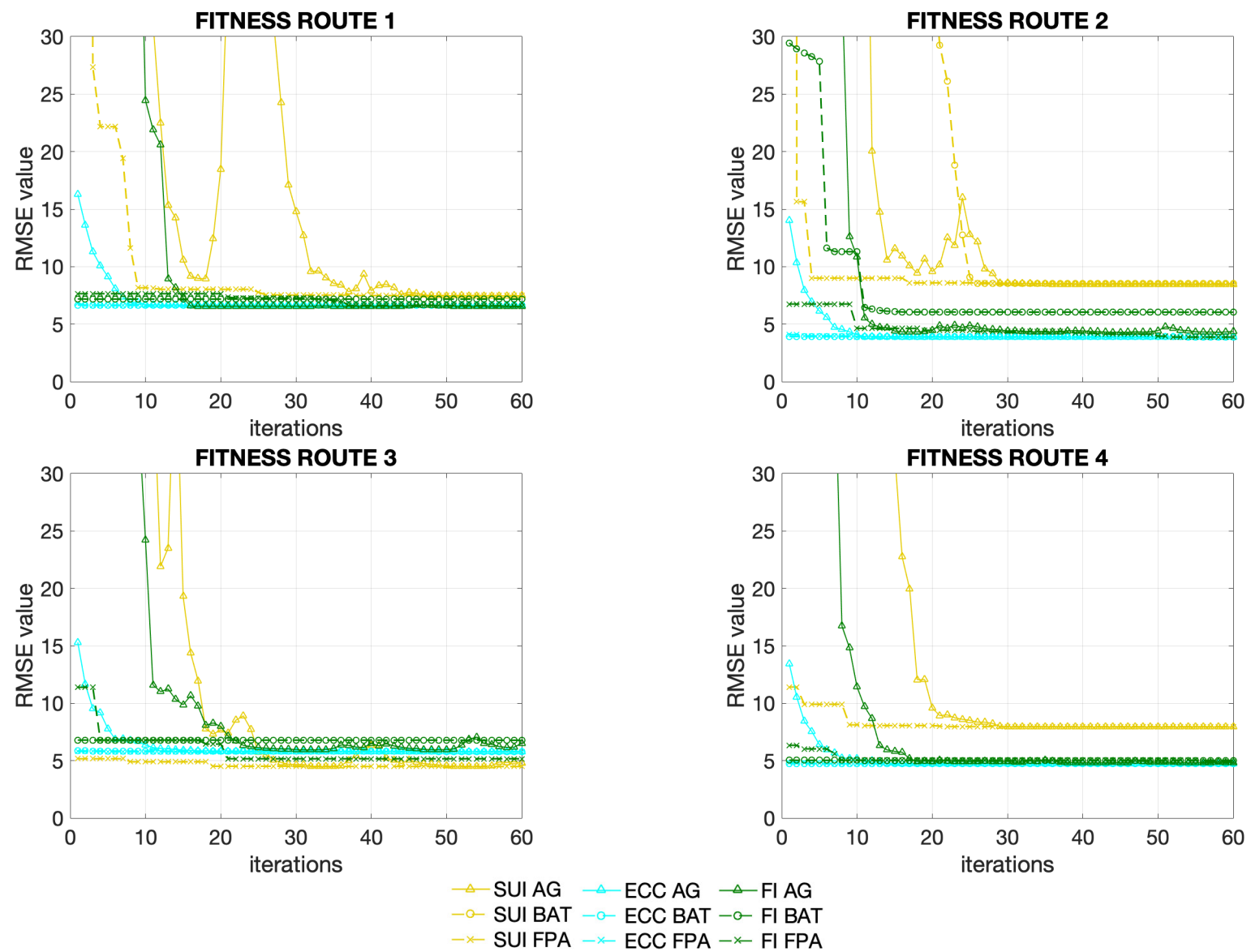

Fig. 8. Plot best fitness.

In Route 2, Fig. 8(b), the AG remains in need of more iterations to achieve the optimal result, since the BAT algorithms converged before the AG, and FPA obtained an optimal result with a minimum amount of iteration.

In Route 3, Fig. 8 (c), the AG has the worst performance, when compared to the other routes, as it converges only close to 30 iterations. BAT achieved a result with the minimum number of iterations required, and the FPA has a similar performance, but it needs about 20 iterations to obtain the optimal result.

Finally, on Route 4, Fig. 8(d), the AG algorithm has a worse performance behavior, as it needs almost 30 iterations to obtain the optimal result. While the BAT algorithm has a performance of approximately 20 iterations to achieve convergence to the optimal solution, and the FPA algorithm requires a minimum of iterations to obtain the optimum result.

It is possible to verify that the AG has the worst performance in all cases when compared to the BAT and FPA algorithms that were selected by this study, considering the execution of the algorithms. However, it is possible to verify that the BAT has a better performance in general when comparing the three algorithms. However, even though the number of iterations does not vary for each algorithm (1.000 iterations), all the algorithms converged to an optimal result and reached similar RMSE values.

\section{CONCLUSION}

This study established a methodology for making adjustments to the classic propagation models designed for wooded habitats. These adjustments were necessary because models like ECC-33, SUI, 
and FI are for different environments in the Amazon region. There was a demonstration that the use of BICs in the adjusted models results in lower RMSE when compared to the classic since the studied environments have trees and buildings that directly interfere in the transmission and reception signal.

In this study, it was possible to identify that for the selected region, the algorithms converge to an ideal solution after adjusting the propagation models. The methodology proposed in this study applies in any similar environment for planning telecommunications systems.

The RMSE values for all routes and modeling showed that the lowest solution found was given in Route 2 of the ECC-33 model with a value of 3.87, a path characterized as a suburban environment with tall buildings and an extensive green corridor. Conversely, the highest RMSE value was on Route 2 in the SUI model with a value of $8.46 \mathrm{~dB}$.

The results obtained in this study demonstrate that the use of forecasting methods through adjustments with BICs, in the planning phase of the communication systems, improves the efficiency and accuracy for the implantation of the base station in several different environments. This solution reflects the decrease in operating costs in these scenarios. Therefore, the proposed technique can be considered a useful tool for LTE and LTE-A wireless network engineers and has proven to be an efficient and accurate modeling methodology.

\section{ACKNOWLEDGMENT}

We would like to thank the Institute of Technology of UFPA and the Laboratory of Computation and Telecommunications. This study was financed in part by the Coordenação de Aperfeiçoamento de Pessoal de Nível Superior - Brasil (CAPES) - Finance Code 001.

\section{REFERENCES}

[1] S. Sirotkin et al., "Lte-wlan aggregation (lwa): Benefits and deployment considerations," White Paper, pp. 1-22, 2016.

[2] C.-C. Huang and W.-C. Lin, "A radio transceiver architecture for coexistence of $4 \mathrm{~g}-\mathrm{LTE}$ and $5 \mathrm{~g}$ systems used in mobile devices," 2017 IEEE MTT-S International Microwave Symposium (IMS), jun 2017.

[3] G. Intelligence, "The mobile economy 2020," Technical Report GSMA.

[4] A. Elnashar and M. A. El-Saidny, "Looking at LTE in practice: A performance analysis of the LTE system based on field test results," IEEE Vehicular Technology Magazine, vol. 8, no. 3, pp. 81-92, sep 2013.

[5] S. F. Chien, C. Zarakovitis, T. O. Ting, and X.-S. Yang, "Bio-inspired approaches in telecommunications," Bio-Inspired Computation in Telecommunications, pp. 23-42, 2015.

[6] M. Feng, L. Guomin, and G. Wenrong, "Heterogeneous network resource allocation optimization based on improved bat algorithm," 2018 International Conference on Sensor Networks and Signal Processing (SNSP), oct 2018.

[7] E. H. Houssein, M. R. Saad, K. Hussain, W. Zhu, H. Shaban, and M. Hassaballah, "Optimal sink node placement in large scale wireless sensor networks based on harris' hawk optimization algorithm,” IEEE Access, vol. 8, pp. 19381-19397, 2020.

[8] T. Rappaport, Wireless Communications: Principles and practice, 3rd, Ed. Prentice-Hall, New-Jersey: Upper Saddle River, 2002.

[9] T. S. Rappaport, Y. Xing, G. R. MacCartney, A. F. Molisch, E. Mellios, and J. Zhang, "Overview of millimeter wave communications for fifth-generation (5g) wireless networks-with a focus on propagation models," IEEE Transactions on Antennas and Propagation, vol. 65, no. 12, pp. 6213-6230, dec 2017.

[10] M. C. A. Neto, J. P. L. Araujo, F. J. B. Barros, A. N. Silva, G. P. S. Cavalcante, and A. G. D. Assuncao, "Bioinspired multiobjective synthesis of x-band FSS via general regression neural network and cuckoo search algorithm," Microwave and Optical Technology Letters, vol. 57, no. 10, pp. 2400-2405, jul 2015.

[11] M. C. de Alcântara Neto, H. R. O. Ferreira, J. P. L. de Araújo, F. J. B. Barros, A. G. Neto, M. de Oliveira Alencar, and G. P. dos Santos Cavalcante, "Compact ultra-wideband FSS optimised through fast and accurate hybrid bio-inspired multiobjective technique," IET Microwaves, Antennas \& Propagation, vol. 14, no. 9, pp. 884-890, jul 2020. 
[12] M. C. A. Neto, J. P. L. Araújo, R. J. S. Mota, F. J. B. Barros, F. H. C. S. Ferreira, G. P. S. Cavalcante, and B. S. L. Castro, "Design and synthesis of an ultra wide band FSS for mm-wave application via general regression neural network and multiobjective bat algorithm," Journal of Microwaves, Optoelectronics and Electromagnetic Applications, vol. 18, no. 4, pp. 530-544, dec 2019.

[13] A. Shahid, S. Aslam, H. S. Kim, and K.-G. Lee, "Component carrier selection method for LTE-advanced using metaheuristic approach," 2013 International Conference on ICT Convergence (ICTC), oct 2013.

[14] M. Shojafar, L. Chiaraviglio, N. Blefari-Melazzi, and S. Salsano, "P5g: A bio-inspired algorithm for the superfluid management of 5g networks," GLOBECOM 2017 - 2017 IEEE Global Communications Conference, dec 2017.

[15] N. Jayanthi and K. R. Valluvan, "Bio-inspired optimization routing technique using DNA sequencing algorithm for wireless sensor networks," Wireless Personal Communications, vol. 101, no. 4, pp. 2365-2381, may 2018.

[16] B. J. Cavalcanti, G. A. Cavalcante, L. M. de Mendonça, G. M. Cantanhede, M. M. de Oliveira, and A. G. D’ Assunção, "A hybrid path loss prediction model based on artificial neural networks using empirical models for LTE and LTE-a at $800 \mathrm{MHz}$ and $2600 \mathrm{MHz}$," Journal of Microwaves, Optoelectronics and Electromagnetic Applications, vol. 16, no. 3, pp. 708-722, sep 2017.

[17] M. Ayadi, A. B. Zineb, and S. Tabbane, "A UHF path loss model using learning machine for heterogeneous networks," IEEE Transactions on Antennas and Propagation, vol. 65, no. 7, pp. 3675-3683, jul 2017.

[18] G. Solutions, "Tools \& toys for radio network planning and optimization," 2010. [Online]. Available: https://www.gyokovsolutions.com/G-NetTrackAndroid.html

[19] S. F. Mjølsnes and R. F. Olimid, "Easy 4g/lte imsi catchers for non-programmers," Computer Network Security, pp. 235-246, 2017.

[20] Yang, Engineering Optimization. John Wiley and Sons, 2010.

[21] A. H. Alavi and A. H. Gandomi, "A robust data mining approach for formulation of geotechnical engineering systems," Engineering Computations, vol. 28, no. 3, pp. 242-274, apr 2011.

[22] Y. Wang, X. Chen, J. Li, and W. Huang, "A genetic-algorithm-based information evolution model for social networks," China Communications, vol. 13, no. 12, pp. 234-249, dec 2016.

[23] S. Sinha and N. Mandal, "Design and analysis of an intelligent flow transmitter using artificial neural network," IEEE Sensors Letters, vol. 1, no. 3, pp. 1-4, jun 2017.

[24] R. Eberhart and J. Kennedy, "A new optimizer using particle swarm theory."

[25] X. S. Yang, "Flower pollination algorithm for global optimization," Unconventional Computation and Natural Computation 2012, Lecture Notes in Computer Science, vol. 7445, 2012.

[26] X.-S. Yang, "A new metaheuristic bat-inspired algorithm," Nature Inspired Cooperative Strategies for Optimization (NICSO 2010), 2010.

[27] X. S. Yang and S. Deb, "Cuckoo search via levy flights," Nature Biologically Inspired Computing, 2009.

[28] Y. Sawaragi, Theory of multiobjective optimization. Orlando: Academic Press, 1985.

[29] C. Blum and A. Roli, "Metaheuristics in combinatorial optimization," ACM Computing Surveys, vol. 35, no. 3, pp. 268-308, sep 2003.

[30] Z. Cui, R. Alex, R. Akerkar, and X.-S. Yang, "Recent advances on bioinspired computation," The Scientific World Journal, vol. 2014, pp. 1-3, 2014.

[31] S. Luke, Essentials of metaheuristics : a set of undergraduate lecture notes. Place of publication not identified: Lulu Com, 2013.

[32] M. Iskander and Z. Yun, "Propagation prediction models for wireless communication systems," IEEE Transactions on Microwave Theory and Techniques, vol. 50, no. 3, pp. 662-673, mar 2002.

[33] V. Erceg, L. Greenstein, S. Tjandra, S. Parkoff, A. Gupta, B. Kulic, A. Julius, and R. Bianchi, "An empirically based path loss model for wireless channels in suburban environments," IEEE Journal on Selected Areas in Communications, vol. 17, no. 7, pp. 1205-1211, jul 1999.

[34] V. E. et al, "Channel models for fixed wireless applications, in ieee 802.16 broadband wireless access working group," July 2001.

[35] Y. Okumura, E. Ohmori, T. Kawano, and K. Fukuda, "Fieldstrength and its variability in vhf and uhf land mobile radio service," 1968.

[36] P. Kyösti, J. Meinilä, L. Hentila, X. Zhao, T. Jämsä, C. Schneider, M. Narandzic, M. Milojević, A. Hong, J. Ylitalo, V.-M. Holappa, M. Alatossava, R. Bultitude, Y. Jong, and T. Rautiainen, "Winner ii channel models," IST-4-027756 WINNER II D1.1.2 V1.2, 022008. 
Journal of Microwaves, Optoelectronics and Electromagnetic Applications, Vol. 20, No. 3, September 2021

DOI: http://dx.doi.org/10.1590/2179-10742021v20i31099

[37] 3rd Generation Partnership Project, "Technical specification group radio acess network: Spatial channel model for multiple input multiple output (mimo)," release 15. [Online]. Available: https://portal.3gpp.org/desktopmodules/ Specifications/SpecificationDetails.aspxspecificationId=1382

[38] M. K. Samimi, T. S. Rappaport, and G. R. MacCartney, "Probabilistic omnidirectional path loss models for millimeterwave outdoor communications," IEEE Wireless Communications Letters, vol. 4, no. 4, pp. 357-360, aug 2015.

[39] G. R. MacCartney, J. Zhang, S. Nie, and T. S. Rappaport, "Path loss models for 5g millimeter wave propagation channels in urban microcells," 2013 IEEE Global Communications Conference (GLOBECOM), dec 2013.

[40] S. Sun, T. S. Rappaport, S. Rangan, T. A. Thomas, A. Ghosh, I. Z. Kovacs, I. Rodriguez, O. Koymen, A. Partyka, and J. Jarvelainen, "Propagation path loss models for 5g urban micro- and macro-cellular scenarios," 2016 IEEE 83rd Vehicular Technology Conference (VTC Spring), may 2016.

[41] E. Ostlin, H.-J. Zepernick, and H. Suzuki, "Macrocell radio wave propagation prediction using an artificial neural network," 60th Vehicular Technology Conference, 2004.

[42] I. Popescu, A. Kanstas, E. Angelou, L. Nafornita, and P. Constantinou, "Applications of generalized RBF-NN for path loss prediction," The 13th IEEE International Symposium on Personal, Indoor and Mobile Radio Communications. 